\title{
Energy-Efficient ID-based Group Key Agreement Protocols for Wireless Networks
}

\author{
Chik How Tan ${ }^{1}$ and Joseph Chee Ming Teo ${ }^{2}$ \\ ${ }^{1}$ NISlab \\ Department of Computer Science \\ ${ }^{2}$ Information Communication Institute of Singapore \\ School of Electrical and Electronic Engineering \\ and Media Technology \\ Gjøvik University College, Norway \\ Nanyang Technological University \\ Singapore 639798 \\ chik.tan@hig.no \\ jose0002@ntu.edu.sg
}

\begin{abstract}
One useful application of wireless networks is for secure group communication, which can be achieved by running a Group Key Agreement (GKA) protocol. One well-known method of providing authentication in GKA protocols is through the use of digital signatures. Traditional certificate-based signature schemes require users to receive and verify digital certificates before verifying the signatures but this process is not required in ID-based signature schemes. In this paper, we present an energy-efficient ID-based authenticated GKA protocol and four energy-efficient ID-based authenticated dynamic protocols, namely Join, Leave, Merge and Partition protocol, to handle dynamic group membership events, which are frequent in wireless networks. We provide complexity and energy cost analysis of our protocols and show that our protocols are more energyefficient and suitable for wireless networks.
\end{abstract}

\section{Introduction}

One useful application of wireless networks is for group communication. However, as the messages in wireless networks are broadcast in plain, they do not provide secure group communication. To provide secure group communication, a group key agreement (GKA) protocol can be used to establish a common group key known only to the users in the group.

The most common way of providing authentication in GKA protocols is through the use of digital signatures. However, this approach usually requires each group member to verify all messages received, which can be large when group size $n$ is large. In certificate- based Public Key Cryptography, before a user can use the public key of the signer to verify the signature, the user has to first obtain and verify the digital certificate issued by a Certifying Authority (CA) to the signer. This results in additional computational cost. ID-based signature schemes do not require the reception and verification of certificates as there are no requirements for public keys in ID-based schemes.

In 1994, Burmester and Desmedt proposed an efficient and secure Burmester-Desmedt (BD) GKA protocol [2] that is suitable for wireless networks. One intuitive way of providing authentication for the BD protocol is to sign and verify all messages sent and received respectively. Although only two signatures are required to be generated, each group member will have to verify $n+2$ messages. The original paper of $\mathrm{BD}$ [2] also did not provide protocols to handle dynamic group membership events such as user join, user leave, network merge and network partition, which occur frequently in wireless networks that have dynamic network topology. One intuitive but inefficient method to handle such events as pointed out in [1] and [10] is to re-execute the $\mathrm{BD}$ protocol.

In this paper, we present an authenticated GKA protocol that is based on a variant of the GuillouQuisquater (GQ) signature scheme [5] and the BD protocol [2]. The GQ signature scheme is an efficient ID-based scheme that is not based on pairing, which has high computational cost [14]. We then compare our scheme with other authenticated versions of the BD protocol and the ID-based Saeednia-Safavi-Naini (SSN) GKA protocol [12], which is also based on the $\mathrm{BD}$ protocol. The complexity and energy cost analysis are based on the $133 \mathrm{MHz}$ "StrongARM" microprocessor and two communication transceivers commonly 
used in wireless networks. From the energy cost analysis, we show that our scheme is the most efficient. Next, we present four authenticated dynamic protocols, namely Join, Leave, Merge and Partition protocol to handle dynamic group membership events. Similarly, we provide a complexity and energy cost analysis of our dynamic protocols with the BD and show that the energy consumed by nodes running our dynamic protocols are significantly lower.

This paper is organized as follows: Section 2 describes the related work in this area of research while a variant of the GQ signature scheme is presented in Section 3. We present our proposed protocol in Section 4 followed by its complexity and energy analysis in Section 5 and 6 respectively. Section 7 presents our four dynamic protocols and their complexity and energy analysis are given in Section 8. Finally we conclude in Section 9.

\section{Related Work}

In 1982, Ingemarsson et al. [7] proposed the first GKA protocol known as ING protocol. Following their work, many GKA protocols such as [15, 2, 10, 4, 12] were proposed. One of these protocols, BurmesterDesmedt (BD) [2] protocol, is an efficient and secure GKA protocol, which Katz and Yung [9] recently provided a rigorous security proof in the standard model. Saeednia and Safavi-Naini [12] proposed an ID-based authenticated GKA protocol (SSN) that is based on the BD in 1998. Although this protocol provides authenticated GKA, the number of exponentiations required to be performed by each user is dependent on the group size $n$. Furthermore, the authors did not specify any dynamic GKA protocols. Currently, most GKA protocols are analyzed based on their complexity. In wireless networks such as MANETs where nodes are usually low power energy constrained devices such as PDAs and sensor nodes, it will be more appropriate to analyze the exact energy consumed by each node for different GKA protocols based on the computational and communication costs.

\section{GQ ID-based Signature Scheme}

Guillou and Quisquater [5] designed an efficient IDbased Signature scheme (GQ) in 1990. We present a variant of the GQ signature scheme as follows:

Setup : The Private Key Generator (PKG) selects two large primes $p^{\prime}$ and $q^{\prime}$ and computes $n=p^{\prime} q^{\prime}$. Next, the PKG chooses a large number $d$ that is relatively prime to $\Phi(n)$, where $\Phi()$ is Euler's totient function and calculates $e$ such that $\operatorname{gcd}(e, d)=1$. The PKG also selects a one way hash function $H:\{0,1\}^{*} \rightarrow\{0,1\}^{l}$, where $l$ is a security parameter. The parameters params are $(n, e, H)$ and the master keys are $\left(p^{\prime}, q^{\prime}, d\right)$.

Extract : The PKG verifies the given user identity $I D$ and computes the secret key for the identity as $S_{I D}=H(I D)^{d} \bmod n$. The secret key $S_{I D}$ is then sent securely to user $I D$.

Sign : Given a private key $S_{I D}$ and a message $M$, choose $\tau \in \in_{R} Z_{n}^{*}$ and compute $c=H\left(\tau^{e}, M\right)$ and $s=$ $\tau \cdot S_{I D}^{c} \bmod n$. Then $\sigma=(s, c)$ is the signature of $M$. Verify : The signature $\sigma=(s, c)$ of an identity $I D$ on a message $M$ is valid if the equation $c=H\left(s^{e}\right.$. $\left.(H(I D))^{-c}, m\right)$ holds good.

\section{Proposed ID-based Authenticated Group Key Agreement Protocol}

The proposed authenticated GKA protocol is IDbased and uses batch verification based on a variant of the GQ signature scheme. We assume that only honest and trusted nodes are participating in the GKA.

Let $G=\left\{U_{1}, \cdots, U_{n}\right\}$ be the initial group of $n$ users where $U_{i}=I D_{i}$ for $i \in\{1, \cdots, n\}$ refers to the given identity of user $U_{i}$. We consider a ring structure among the users of $G$ where the users' indices can be considered on the circulation of $\{1, \cdots, n\}$. The proposed protocol consist of two rounds during which each user $U_{i}$ will broadcast their key materials $z_{i}$ and $X_{i}$ in Round 1 and Round 2 respectively for group key computation. The protocol works as follows:

Setup : The PKG first selects two large (512-bit) prime $\overline{\text { numbers }} p^{\prime}$ and $q^{\prime}$ and calculates $n=p^{\prime} q^{\prime}$. Next, the PKG chooses a large number $d$ that is relatively prime to $\Phi(n)$ and calculates $e$ such that $\operatorname{gcd}(e, d)=1$. The PKG also selects two random large primes, $q$ (160-bit) and $p$ (1024-bit), such that $q$ divides $p-1$ for the GKA protocol. Next, an element $g \in Z_{p}^{*}$ of order $q$ is selected as the generator. Finally, the PKG selects a one way hash function $H:\{0,1\}^{*} \rightarrow\{0,1\}^{l}$, where $l$ is a security parameter. The parameters params are $(n, e, p, q, g, H)$ and the master keys are $\left(p^{\prime}, q^{\prime}, d\right)$.

Extract : The PKG verifies the 32-bit identity $U_{i}=$ $I D_{i}$ of user $U_{i}$ and computes the secret key for $U_{i}$ as $S_{U_{i}}=H\left(U_{i}\right)^{d} \bmod n$. The secret key $S_{U_{i}}$ is then sent securely to $U_{i}$.

$\underline{\text { Round } 1}$ : Each user $U_{i}$ for $i \in\{1, \cdots, n\}$ first selects $r_{i} \in Z_{q}^{*}$ and $\tau_{i} \in Z_{n}^{*}$ and computes $z_{i}=g^{r_{i}} \bmod p$ and $t_{i}=\tau_{i}^{e} \bmod n$. $U_{i}$ then broadcast $m_{i}=U_{i}\left\|z_{i}\right\| t_{i}$ (where $\|$ denotes concatenation of messages).

$\underline{\text { Round } 2}$ : Each user $U_{i}$ first computes :

$$
X_{i}=\left(\frac{z_{i+1}}{z_{i-1}}\right)^{r_{i}}=g^{r_{i} r_{i+1}-r_{i-1} r_{i}} \bmod p
$$


Next, $U_{i}$ computes $\mathcal{Z}=\prod_{i=1}^{n} z_{i} \bmod p, \quad \mathcal{T}=$ $\prod_{i=1}^{n} t_{i} \bmod n$ and $c=H(\mathcal{T}, \mathcal{Z}) . \quad U_{i}$ then computes $s_{i}=\tau_{i} \cdot S_{U_{i}}^{c} \bmod n$ and produces the signature $\sigma_{i}=\left(s_{i}, c\right) . \quad U_{i}$ stores $\mathcal{Z}$ and $c$ and broadcasts $m_{i}^{\prime}=U_{i}\left\|X_{i}\right\| s_{i}$. It is noted that $U_{1}$ is assumed to be a trusted controller such that $U_{1}$ will be the last user to broadcast its message $m_{1}^{\prime}$ after all the other users $U_{j} \neq U_{1}$ have broadcast their messages $m_{j}^{\prime}$.

Authentication and Key Computation: Each $U_{i}$ verifies the received messages $m_{j}^{\prime} \neq m_{i}^{\prime}$ by $u$ sing the stored $\mathcal{Z}$ and $c$ to check the equation:

$$
c=H\left(\left(\prod_{i=1}^{n} s_{i}\right)^{e} \cdot\left(\prod_{i=1}^{n} H\left(U_{i}\right)\right)^{-c}, \mathcal{Z}\right)
$$

If equation (2) is correct, $U_{i}$ will proceed to verify the $X_{i}$ values broadcast in Round 2 . However, if equation (2) is incorrect, then all members will retransmit again. From equation (1), we have $X_{i}=$ $g^{r_{i} r_{i+1}-r_{i-1} r_{i}} \bmod p$ for $i \in\{1, \ldots, n\}$, where $r_{0}=r_{n}$ and $r_{n+1}=r_{1}$. By simple computation, we have the following lemma:

Lemma $1 \prod_{i=1}^{n} X_{i}=1 \bmod p$.

Using Lemma 1, we can check whether the $X_{i}$ sent by group member $U_{i}$ is genuine. If $\prod_{i=1}^{n} X_{i} \neq 1 \bmod p$, it means that at least one of the $X_{i}$ is incorrect. Then, all members will retransmit again. If each $U_{i}$ correctly verifies that $\prod_{i=1}^{n} X_{i}=1 \bmod p$, then $U_{i}$ computes the common group key $K$ as follows:

$$
K=\prod_{i=1}^{n} g^{r_{i} r_{i+1}} \bmod p=g^{r_{1} r_{2}+\cdots+r_{n} r_{1}} \bmod p(3)
$$

where $r_{0}=r_{n}$ and $r_{n+1}=r_{1}$.

\section{Complexity Analysis}

Table 1 presents the complexity analysis of different protocols to achieve authenticated BD and the Saeednia-Safavi-Naini (SSN) scheme [12]. The first protocol refers to our proposed GKA scheme in Section 4. The second protocol uses 194-bit ID-based SOK signature scheme [13] to provide authentication for BD. The third and fourth protocols are the BD with 160-bit ECDSA and BD with 1024-bit DSA signature scheme respectively. The last protocol, 1024-bit SSN scheme, uses ID-based cryptography. Although the last protocol do not require any signature generation and verifications, the number of modular exponentiations required is $2 n+4$. All other protocols requires only three modular exponentiations for the BD GKA protocol.
Table 1. Complexity Analysis for Authenticated BD GKA

\begin{tabular}{||c|c|c|c|c|c||}
\hline \hline & $\begin{array}{c}\text { Our } \\
\text { Prop. } \\
\text { sch. }\end{array}$ & $\begin{array}{c}\text { BD } \\
\text { with } \\
\text { SOK }\end{array}$ & $\begin{array}{c}\text { BD } \\
\text { with } \\
\text { ECDSA }\end{array}$ & $\begin{array}{c}\text { BD } \\
\text { with } \\
\text { DSA }\end{array}$ & $\begin{array}{c}\text { SSN } \\
\text { sch. }\end{array}$ \\
\hline Exp. & 3 & 3 & 3 & 3 & $a$ \\
\hline Msg Tx & 2 & 2 & 2 & 2 & 2 \\
\hline Msg Rx & $b$ & $b$ & $b$ & $b$ & $b$ \\
\hline Cert Tx & - & - & 1 & 1 & - \\
\hline Cert Rx & - & - & $n-1$ & $n-1$ & - \\
\hline Cert Ver & - & - & $n-1$ & $n-1$ & - \\
\hline MapToPt & - & $n-1$ & - & - & - \\
\hline Sign Gen & 1 & 1 & 1 & 1 & - \\
\hline Sign Ver & 1 & $n-1$ & $n-1$ & $n-1$ & - \\
\hline \hline
\end{tabular}

$a: 2 n+4 \quad b: 2(n-1)$

All protocols, including SSN scheme, require two message transmission and $2(n-1)$ messages to be received by each user. The third and fourth protocol require each user to transmit their certificate in the first message as well as receive and verify $n-1$ certificates from other users. The second protocol is based on pairing and requires $n-1$ MapToPoint operations.

All protocols except the SSN scheme requires one signature generation. This signature generation is done in Round 2 of the GKA for the second, third and fourth protocol where each user signs the message $m_{i}=U_{i}\left\|z_{i}\right\| X_{i} \| \prod_{i=1}^{n} z_{i}$ to provide authentication for both keying materials $z_{i}$ and $X_{i}$ broadcast in Rounds 1 and 2 of the BD respectively. In terms of signature verifications, our proposed protocol is the most efficient.

\section{$6 \quad$ Energy Analysis}

In this section, we perform the total energy consumption cost analysis of performing authenticated BD using the $133 \mathrm{MHz}$ SA-1110 "StrongARM" microprocessor and two different communication transceivers, namely the $100 \mathrm{kbps}$ radio transceiver module and the IEEE 802.11 Spectrum24 WLAN card. We then present a graph that illustrates the total energy consumed by each node while performing authenticated GKA using the protocols shown in Table 1 with the "StrongARM" microprocessor and the two transceiver modules for group size $n=10,50,100$ and 500 .

\section{Computational Energy Cost}

Table 2 shows the computational energy consumption costs, the computational timing costs of the $133 \mathrm{MHz}$ "StrongARM" microprocessor and the computational timing costs of the Pentium III $450 \mathrm{MHz}$ 
Table 2. Computational Energy Cost

\begin{tabular}{|c|c|c|c|c|}
\hline \multirow{2}{*}{\multicolumn{2}{|c|}{ Mod. }} & \multicolumn{2}{|c|}{$\begin{array}{c}133 \mathrm{MHz} \\
\text { StrongARM }\end{array}$} & $\begin{array}{c}450 \mathrm{MHz} \\
\text { P-III }\end{array}$ \\
\hline & & $\overline{9.1 m J}$ & $37.92 \mathrm{~ms}$ & $8.8 \mathrm{~ms}$ \\
\hline \multicolumn{2}{|c|}{ MapToPoint } & $18.4 m J$ & $76.67 \mathrm{~ms}$ & $17.78 \mathrm{~ms}$ \\
\hline \multicolumn{2}{|c|}{ Tate Pairing } & $47.0 \mathrm{~mJ}$ & $191.5 \mathrm{~ms}$ & $44.4 \mathrm{~ms}$ \\
\hline \multicolumn{2}{|c|}{ Scalar Mul. } & $8.8 \mathrm{~mJ}$ & $36.67 \mathrm{~ms}$ & $8.5 \mathrm{~ms}$ \\
\hline \multirow{4}{*}{$\begin{array}{l}\text { Sign. } \\
\text { Gen. }\end{array}$} & DSA & $9.1 \mathrm{~mJ}$ & $37.92 \mathrm{~ms}$ & $8.8 \mathrm{~ms}$ \\
\hline & ECDSA & $8.8 m J$ & $36.67 \mathrm{~ms}$ & $8.5 \mathrm{~ms}$ \\
\hline & SOK & $17.6 \mathrm{~mJ}$ & $73.33 \mathrm{~ms}$ & $17 \mathrm{~ms}$ \\
\hline & GQ & $18.2 \mathrm{~mJ}$ & $75.83 \mathrm{~ms}$ & $17.6 \mathrm{~ms}$ \\
\hline \multirow{4}{*}{$\begin{array}{l}\text { Sign. } \\
\text { Ver. }\end{array}$} & DSA & $11.1 \mathrm{~mJ}$ & $46.33 \mathrm{~ms}$ & $10.75 \mathrm{~ms}$ \\
\hline & ECDSA & $10.9 \mathrm{~mJ}$ & $45.42 \mathrm{~ms}$ & $10.5 \mathrm{~ms}$ \\
\hline & SOK & $137.7 \mathrm{~mJ}$ & $573.75 \mathrm{~ms}$ & $133.2 \mathrm{~ms}$ \\
\hline & GQ & $18.2 m J$ & $75.83 \mathrm{~ms}$ & $17.6 \mathrm{~ms}$ \\
\hline
\end{tabular}

microprocessor $(\mathrm{P} 3-450 \mathrm{MHz})$ for performing different cryptographic and signature operations.

From [3], we obtained the energy consumption cost of modular exponentiation for the "StrongARM" microprocessor to be $9.1 \mathrm{~mJ}$. As the "StrongARM" microprocessor power consumption is $240 \mathrm{~mW}$ [3], we can obtain the timing cost of modular exponentiation to be $\frac{9.1 \mathrm{~mJ}}{240 \mathrm{~mW}}=37.92 \mathrm{~ms}$. Based on the MIRACL software library [11], we obtained the computational timing costs of modular exponentiation $(8.8 \mathrm{~ms})$ and other cryptographic operations executed on a Pentium III $450 \mathrm{MHz}$ (P3-450MHz) microprocessor in Table 3. From these information, we can extrapolate and estimate the time taken $\alpha(m s)$ for primitive cryptographic operation Y (e.g. Tate Pairing) on the "StrongARM" microprocessor as follows:

$$
\alpha=\frac{\gamma \mathrm{ms}}{8.8 \mathrm{~ms}} \times 37.92 \mathrm{~ms}
$$

where $\gamma$ refers to the time taken for primitive operation $\mathrm{Y}$ on the $\mathrm{P} 3-450 \mathrm{MHz}$ microprocessor. Next, we estimate the energy consumed $\beta(m J)$ for primitive operation Y on the $133 \mathrm{MHz}$ "StrongARM" microprocessor as $\beta=240 \mathrm{~m} W \times \alpha \mathrm{ms}$. With these information, we obtained the energy costs in Table 2. The timing cost of the Tate Pairing operation was given to be $20 \mathrm{~ms}$ on the Pentium III $1 \mathrm{GHz}$ (P3-1GHz) microprocessor [11]. To obtain the equivalent timing cost on the P3$450 \mathrm{MHz}$ microprocessor, we scale down by a factor of $\frac{1000 \mathrm{MHz}}{450 \mathrm{MHz}}=2.22$ to obtain $44.4 \mathrm{~ms}$. From [11], the timing costs of Identity-based Encryption (IBE) Encrypt and Decrypt were given to be $35 \mathrm{~ms}$ and $27 \mathrm{~ms}$ respectively on the P3-1GHz. As the IBE Encrypt requires one additional MapToPoint operation than IBE De-
Table 3. Communication Energy Cost

\begin{tabular}{||c|c|c||}
\hline \hline & $\begin{array}{c}\text { 100kbps } \\
\text { Transceiver }\end{array}$ & $\begin{array}{c}\text { WLAN } \\
\text { Card }\end{array}$ \\
\hline Tx per bit & $10.8 \mu J$ & $0.66 \mu J$ \\
\hline Rx per bit & $7.51 \mu J$ & $0.31 \mu J$ \\
\hline Tx. 263-Bytes DSA cert & $22.72 m J$ & $1.38 m J$ \\
\hline Rx. 263-Bytes DSA cert & $15.8 m J$ & $0.64 m J$ \\
\hline Tx. 86-Bytes ECDSA cert & $7.43 m J$ & $0.45 m J$ \\
\hline Rx. 86-Bytes ECDSA cert & $5.17 m J$ & $0.21 m J$ \\
\hline Tx. DSA/ECDSA sign. $^{1}$ & $3.46 m J$ & $0.21 m J$ \\
\hline Rx. DSA/ECDSA sign. ${ }^{1}$ & $2.40 m J$ & $0.1 m J$ \\
\hline Tx. SOK sign. $^{2}$ & $4.19 m J$ & $0.26 m J$ \\
\hline Rx. SOK sign. $^{2}$ & $2.91 m J$ & $0.12 m J$ \\
\hline Tx. GQ sign. ${ }^{3}$ & $12.79 m J$ & $0.78 m J$ \\
\hline Rx. GQ sign. ${ }^{3}$ & $8.89 m J$ & $0.36 m J$ \\
\hline \hline
\end{tabular}

${ }^{1}$ DSA/ECDSA signature $(r, s)$, both $r, s=160$-bits.

2 SOK signature $\left(S_{1}, S_{2}\right)$, both $S_{1}, S_{2}=194$-bits.

${ }^{3}$ GQ signature $(s, c), s=1024$-bits and $c=160$-bits.

crypt, we were able to obtain the timing cost of MapToPoint operation to be $35-27=8 \mathrm{~ms}$. To get the timing cost of the MapToPoint operation on the P3-450MHz, we scale down by a factor of 2.22 to get $17.78 \mathrm{~ms}$.

\section{Communications Energy Cost}

Table 3 shows the communication energy costs comparison using the $100 \mathrm{kbps}$ transceiver module and the IEEE 802.11 Spectrum24 LA-4121 WLAN card. Using the information given in [3] and [6] for the 100kbps transceiver module and [8] for the WLAN card, we obtained the transmission and reception cost per bit of the two transceiver modules. We then obtained the transmission and reception energy costs of transmitting and receiving certificates and signatures.

\section{Energy Consumption Cost Results}

By considering the complexity in Table 1 and the energy costs in Tables 2 and 3, we obtained the graph in Figure 1 that shows the total energy consumption costs of each node using the $133 \mathrm{MHz}$ "StrongARM" microprocessor with either $100 \mathrm{kbps}$ transceiver module or WLAN card. The total energy costs include the transmission and reception costs of all messages as well as the total computational costs in each respective GKA protocol. The figure clearly shows that our proposed scheme is the most energy-efficient when using either the 100kbps transceiver module (i) or WLAN card (j). 


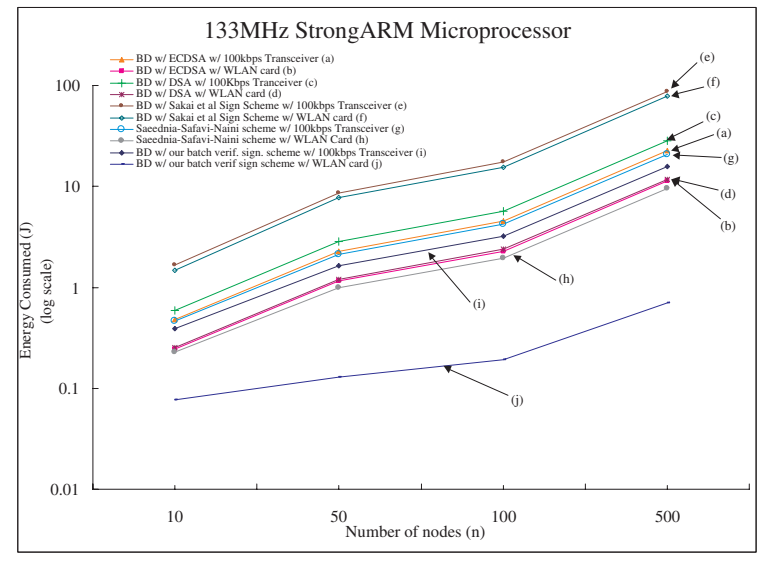

Figure 1. Energy Consumption Costs.

\section{Dynamic Protocols}

In this section, we present four authenticated dynamic protocols, namely the Join, Leave, Merge and Partition protocol, that can be used to efficiently handle dynamic group membership events. We assume that all group members taking part in the dynamic protocols with possession of the current group key $K$ are trusted nodes. Our proposed dynamic protocols use symmetric key cryptography, which studies [3] and [6] have shown to have energy requirements of orders of magnitude lower than modular exponentiations.

\section{Join Protocol}

Let $G=\left\{U_{1}, \cdots, U_{n}\right\}$ be the current group and $U_{n+1}$ be the new user joining the group. We divide $G$ into two parts, $\left\{U_{1}, U_{n}\right\}$, which has the two users actively involved in the Join Protocol and $\left\{U_{2}, \cdots, U_{n-1}\right\}$, which consists of the rest of the group. We consider a ring structure among the users of $G$ with $U_{n+1}$ joining $G$ in between $U_{n}$ and $U_{1}$ to form the new group $G^{\prime}=\left\{U_{1}, \cdots, U_{n+1}\right\}$. The Join Protocol consists of three rounds and works as follows:

Round 1: The new node $U_{n+1}$ first chooses a random $r_{n+1} \bmod q$ and computes $z_{n+1}=g^{r_{n+1}} \bmod p$. Next, $U_{n+1}$ signs $U_{n+1} \| z_{n+1}$ using the variant of the GQ signature scheme (Section III) to obtain the signature $\sigma_{n+1}$ and broadcasts $m_{n+1}=U_{n+1}\left\|z_{n+1}\right\| \sigma_{n+1}$.

Round 2:

(1) $U_{1}$ first verifies the signature $\sigma_{n+1}$. Next, $U_{1}$ chooses a new random $r_{1}^{\prime} \in Z_{q}^{*}$ and computes $K^{*}$ as follows:

$$
\begin{aligned}
K^{*} & =K \cdot\left(z_{2} \cdot z_{n}\right)^{-r_{1}} \cdot\left(z_{2} \cdot z_{n+1}\right)^{r_{1}^{\prime}} \bmod p \\
& =g^{r_{1}^{\prime} r_{2}+\cdots+r_{n-1} r_{n}+r_{n+1} r_{1}^{\prime}} \bmod p .
\end{aligned}
$$

$U_{1}$ then encrypts $K^{*} \| U_{1}$ using the current group key
$K$ and a symmetric key encryption $E_{k}(m)$ (where $m$ is the message for encryption and $k$ is the secret key) to obtain $E_{K}\left(K^{*} \| U_{1}\right)$ and broadcasts $m_{1}^{\prime}=$ $U_{1} \| E_{K}\left(K^{*} \| U_{1}\right)$ to current group $G$.

(2) $U_{n}$ verifies the signature $\sigma_{n+1}$. $U_{n}$ then computes the DH key $K_{U_{n} U_{n+1}}=g^{r_{n} r_{n+1}}=\left(z_{n+1}\right)^{r_{n}} \bmod$ $p$, which it shares with $U_{n+1}$. Next, $U_{n}$ encrypts $K_{U_{n} U_{n+1}} \| U_{n}$ using $K$ to obtain $E_{K}\left(K_{U_{n} U_{n+1}} \| U_{n}\right) . U_{n}$ then signs $E_{K}\left(K_{U_{n} U_{n+1}} \| U_{n}\right) \| z_{n}$ using the variant of the GQ signature scheme to obtain the signature $\sigma_{n}^{\prime \prime}$ and broadcasts $m_{n}^{\prime \prime}=U_{n}\left\|E_{K}\left(K_{U_{n} U_{n+1}} \| U_{n}\right)\right\| z_{n} \| \sigma_{n}^{\prime \prime}$.

$\underline{\text { Round } 3}$ :

(1) $U_{n+1}$ first verifies the signature $\sigma_{n}^{\prime \prime}$. Next, $U_{n+1}$ computes $K_{U_{n} U_{n+1}}=g^{r_{n} r_{n+1}}=\left(z_{n}\right)^{r_{n+1}} \bmod p$.

(2) $U_{n}$ first decrypts $E_{K}\left(K^{*} \| U_{1}\right)$ in $m_{1}^{\prime}$ to obtain $K^{*}$ and the identity $U_{1}$. $U_{n}$ then checks if the identity $U_{1}$ is decrypted correctly to ensure the validity of $K^{*}$. Next, $U_{n}$ encrypts $K^{*} \| U_{n}$ using $K_{U_{n} U_{n+1}}$ to obtain $E_{K_{U_{n} U_{n+1}}}\left(K^{*} \| U_{n}\right)$ and transmits $m_{n}^{\prime \prime \prime}=$ $U_{n} \| E_{K_{U_{n} U_{n+1}}}\left(K^{*} \| U_{n}\right)$ to $U_{n+1}$.

Key Computation :

(1) $U_{n+1}$ decrypts $E_{K_{U_{n} U_{n+1}}}\left(K^{*} \| U_{n}\right)$ in $m_{n}^{\prime \prime \prime}$ to obtain $K^{*}$ and the identity $U_{n} \cdot U_{n+1}^{n+1}$ checks if the identity $U_{n}$ was decrypted correctly to ensure the validity of $K^{*}$.

(2) All users $U_{i} \neq U_{1}, U_{n}$ for $i \in\{2, \cdots, n-1\}$ decrypts $E_{K}\left(K^{*} \| U_{1}\right)$ from $m_{1}^{\prime}$ and $E_{K}\left(K_{U_{n} U_{n+1}} \| U_{n}\right)$ from $m_{n}^{\prime \prime}$ using the current group key $K$ to obtain $K^{*}$, the identity $U_{1}, K_{U_{n} U_{n+1}}$ and the identity $U_{n}$ respectively. Next, each $U_{i}$ checks that the identities $U_{1}$ and $U_{n}$ are decrypted correctly to ensure the validity of $K^{*}$ and $K_{U_{n} U_{n+1}}$.

(3) Finally, all users including $U_{n+1}$ compute the new key $K^{\prime}$ as follows:

$$
\begin{aligned}
K^{\prime} & =K^{*} \cdot K_{U_{n} U_{n+1}} \bmod p \\
& =g^{r_{1}^{\prime} r_{2}+\cdots+r_{n} r_{n+1}+r_{n+1} r_{1}^{\prime}} \bmod p
\end{aligned}
$$

\section{Merge Protocol}

A merge occurs when two or more subgroups are combined into a single group. Let $G_{A}=\left\{U_{1}, \cdots, U_{n}\right\}$ denote all users in Group A with group key $K_{A}$ and $G_{B}=\left\{U_{n+1}, U_{n+2}, \cdots, U_{n+m}\right\}$ denote all users in Group B with group key $K_{B}$. We divide $G_{A}$ into two parts, $\left\{U_{1}, U_{n}\right\}$ and $\left\{U_{2}, \cdots, U_{n-1}\right\}$ and $G_{B}$ into $\left\{U_{n+1}, U_{n+m}\right\}$ and $\left\{U_{n+2}, \cdots, U_{n+m-1}\right\}$. We consider a ring structure for $G_{A}, G_{B}$ and the merged group $G^{\prime}=G_{A} \cup G_{B}=\left\{U_{1}, \cdots, U_{n+m}\right\}$. The Merge Protocol consists of three rounds and works as follows:

Round 1 :

(1) $U_{1}$ first selects a new random $r_{1}^{\prime} \in Z_{q}^{*}$ and computes $\tilde{z}_{1}=g^{r_{1}^{\prime}} \bmod p$. Next, $U_{1}$ signs $U_{1}\left\|\tilde{z}_{1}\right\| z_{n}$ using the variant of the GQ signature scheme to produce the signature $\sigma_{1}^{\prime}$ and broadcasts $m_{1}^{\prime}=U_{1}\left\|\tilde{z}_{1}\right\| z_{n} \| \sigma_{1}^{\prime}$. 
(2) $U_{n+1}$ selects new random $r_{n+1}^{\prime} \in Z_{q}^{*}$, computes $\tilde{z}_{n+1}=g^{r_{n+1}^{\prime}} \bmod p$ and signs $U_{n+1}\left\|\tilde{z}_{n+1}\right\| z_{n+m}$ using the variant of the GQ signature scheme to obtain the signature $\sigma_{n+1}^{\prime}$ and broadcasts $m_{n+1}^{\prime}=$ $U_{n+1}\left\|\tilde{z}_{n+1}\right\| z_{n+m} \| \sigma_{n+1}^{\prime}$.

$\underline{\text { Round } 2}$ :

(1) $U_{1}$ verifies the signature $\sigma_{n+1}^{\prime}$. Next, $U_{1}$ extracts $\tilde{z}_{n+1}$ from $m_{n+1}^{\prime}$ and computes the DH key $K_{U_{1} U_{n+1}}=$ $g^{r_{1}^{\prime} r_{n+1}^{\prime}}=\left(\tilde{z}_{n+1}\right)^{r_{1}^{\prime}} \bmod p$ to be shared with $U_{n+1} \cdot U_{1}$ then computes:

$$
\begin{aligned}
K_{A}^{*} & =K_{A} \cdot\left(z_{2} \cdot z_{n}\right)^{-r_{1}} \cdot\left(z_{2} \cdot z_{n+m}\right)^{r_{1}^{\prime}} \\
& =g^{r_{1}^{\prime} r_{2}+\cdots+r_{n-1} r_{n}+r_{n+m} r_{1}^{\prime}} \bmod p
\end{aligned}
$$

Next, $U_{1}$ encrypts $K_{A}^{*} \| U_{1}$ using Group $A$ 's current key $K_{A}$ and $K_{U_{1} U_{n+1}}$ to obtain $E_{K_{A}}\left(K_{A}^{*} \| U_{1}\right)$ and $E_{K_{U_{1} U_{n+1}}}\left(K_{A}^{*} \| U_{1}\right)$ respectively. Finally, $U_{1}$ broadcasts the message $m_{1}^{\prime \prime}=U_{1}\left\|E_{K_{A}}\left(K_{A}^{*} \| U_{1}\right)\right\| E_{K_{U_{1} U_{n+1}}}\left(K_{A}^{*} \| U_{1}\right)$.

(2) $U_{n+1}$ first verifies the signature $\sigma_{1}^{\prime}$. Next, $U_{n+1}$ extracts $\tilde{z}_{1}$ from $m_{1}^{\prime}$ and computes $K_{U_{1} U_{n+1}}=g^{r_{1}^{\prime} r_{n+1}^{\prime}}=$ $\left(\tilde{z}_{1}\right)^{r_{n+1}^{\prime}} \bmod p$, the DH key shared with $U_{1} \cdot U_{n+1}$ then computes $K_{B}^{*}$ :

$$
\begin{aligned}
K_{B}^{*} & =K_{B} \cdot\left(z_{n} \cdot z_{n+2}\right)^{r_{n+1}^{\prime}} \cdot\left(z_{n+2} \cdot z_{n+m}\right)^{-r_{n+1}} \\
& =g^{r_{n} r_{n+1}^{\prime}+\cdots+r_{n+m-1} r_{n+m}} \bmod p
\end{aligned}
$$

Next, $U_{n+1}$ encrypts $K_{B}^{*} \| U_{n+1}$ using Group $B$ 's current key $K_{B}$ and $K_{U_{1} U_{n+1}}$ to obtain $E_{K_{B}}\left(K_{B}^{*}|| U_{n+1}\right)$ and $E_{K_{U_{1} U_{n+1}}}\left(K_{B}^{*}|| U_{n+1}\right)$ respectively. Finally, $U_{n+1}$ broadcasts $m_{n+1}^{\prime \prime}=$ $U_{n+1}\left\|E_{K_{B}}\left(K_{B}^{*}|| U_{n+1}\right)\right\| E_{K_{U_{1} U_{n+1}}}\left(K_{B}^{*}|| U_{n+1}\right)$.

Round 3 :

(1) Group $B$ users $U_{j} \neq U_{n+1}$ for $j \in\{n+2, \cdots, n+m\}$ first decrypts $E_{K_{B}}\left(K_{B}^{*} \| U_{n+1}\right)$ to obtain $K_{B}^{*}$ and the identity $U_{n+1}$. Next, each $U_{j}$ checks if the identity $U_{n+1}$ was decrypted correctly to ensure that $K_{B}^{*}$ is valid and stores $K_{B}^{*}$ for key computation later.

(2) $U_{1}$ decrypts $E_{K_{U_{1} U_{n+1}}}\left(K_{B}^{*} \| U_{n+1}\right)$ in message $m_{n+1}^{\prime \prime}$ to obtain $K_{B}^{*}$ and the identity $U_{n+1}$. Next, $U_{1}$ checks if the identity $U_{n+1}$ was decrypted correctly to ensure the validity of $K_{B}^{*}$. $\quad U_{1}$ then encrypts $K_{B}^{*} \| U_{1}$ using Group $A$ 's key $K_{A}$ to obtain $E_{K_{A}}\left(K_{B}^{*} \| U_{1}\right)$. Finally, $U_{1}$ broadcasts $m_{1}^{\prime \prime \prime}=U_{1} \| E_{K_{A}}\left(K_{B}^{*} \| U_{1}\right)$ to $G_{A}$.

(3) $U_{n+1}$ decrypts $E_{K_{U_{1} U_{n+1}}}\left(K_{A}^{*} \| U_{1}\right)$ in message $m_{1}^{\prime \prime}$ to obtain $K_{A}^{*}$ and the identity $U_{1}$. Next, $U_{n+1}$ checks if the identity $U_{1}$ was decrypted correctly to ensure that $K_{A}^{*}$ is valid. $U_{n+1}$ then encrypts $K_{A}^{*} \| U_{n+1}$ using Group $B$ 's key $K_{B}$ to obtain $E_{K_{B}}\left(K_{A}^{*} \| U_{n+1}\right)$. Finally, $U_{n+1}$ broadcasts $m_{n+1}^{\prime \prime \prime}=U_{n+1} \| E_{K_{B}}\left(K_{A}^{*} \| U_{n+1}\right)$ to $G_{B}$.

Key Computation :
(1) Group $A$ users $U_{i} \neq U_{1}$ for $i \in\{2, \cdots, n\}$ first decrypts $E_{K_{A}}\left(K_{A}^{*} \| U_{1}\right)$ in message $m_{1}^{\prime \prime}$ and $E_{K_{A}}\left(K_{B}^{*} \| U_{1}\right)$ in $m_{1}^{\prime \prime \prime}$ to obtain $K_{A}^{*}, K_{B}^{*}$ and the identity $U_{1}$. Each $U_{i}$ then verifies that the identity $U_{1}$ decrypted from both messages $m_{1}^{\prime \prime}$ and $m_{1}^{\prime \prime \prime}$ is correct to ensure the validity of $K_{A}^{*}$ and $K_{B}^{*}$ respectively.

(2) Group $B$ users $U_{j} \neq U_{n+1}$ for $j \in\{n+2, \cdots, n+m\}$ first decrypts $E_{K_{B}}\left(K_{A}^{*} \| U_{n+1}\right)$ in $m_{n+1}^{\prime \prime \prime}$ to obtain $K_{A}^{*}$ and $U_{n+1}$. Each $U_{j}$ then verifies that the identity $U_{1}$ decrypted is correct to ensure the received $K_{A}^{*}$ is valid. (3) Finally, all users in the merged group $G^{\prime}$ compute the new group key $K^{\prime}$ as follows:

$$
\begin{aligned}
K^{\prime} & =K_{A}^{*} \cdot K_{B}^{*} \bmod p \\
& =g^{r_{1}^{\prime} r_{2}+\cdots+r_{n} r_{n+1}^{\prime}+\cdots+r_{n+m} r_{1}^{\prime}} \bmod p .
\end{aligned}
$$

\section{Leave Protocol}

Let $G=\left\{U_{1}, \cdots, U_{n}\right\}$ denote the current group and $G^{\prime}=G \backslash U_{l}$ denotes the new group. We also consider a ring structure for $G$ and $G^{\prime}$. The Leave Protocol comprises of two rounds and works as follows:

$\underline{\text { Round } 1}$ :All remaining odd-indexed users $U_{j} \neq U_{l}$ for $j \in\{1,3,5, \cdots\}$ select new randoms $r_{j}^{\prime} \in Z_{q}^{*}$ and $\bar{\tau}_{j} \in$ $Z_{n}^{*}$ and computes $z_{j}^{\prime}=g^{r_{j}^{\prime}} \bmod p$ and $t_{j}^{\prime}=\bar{\tau}_{j}^{e} \bmod n$. $U_{j}$ then broadcasts $m_{j}=U_{j}\left\|z_{j}^{\prime}\right\| t_{j}^{\prime}$.

Round 2 : All users $U_{i} \neq U_{l}$ compute $X_{i}^{\prime}$ as follows:

$$
X_{i}^{\prime}=\left\{\begin{array}{l}
\left(\frac{z_{i+1}}{z_{i-1}}\right)^{r_{i}} \text { if } i \neq l, l-1, l+1, \\
\left(\frac{z_{l+1}}{z_{l-2}}\right)^{r_{l-1}} \text { if } i=l-1, \\
\left(\frac{z_{l+2}}{z_{l-1}}\right)^{r_{l+1}} \text { if } i=l+1 .
\end{array}\right.
$$

Next, each $U_{i} \in G^{\prime}$ computes $\overline{\mathcal{Z}}=\prod_{i=1}^{n} z_{i} \bmod p, \overline{\mathcal{T}}=$ $\prod_{i=1}^{n} t_{i} \bmod n$ (where $z_{i}=z_{j}^{\prime}$ and $t_{i}=t_{j}^{\prime}$ for remaining odd-indexed users $\left.U_{j} \in G^{\prime}\right)$ and $\bar{c}=H(\overline{\mathcal{T}}, \overline{\mathcal{Z}}) . U_{i} \neq U_{l}$ then computes $\bar{s}_{i}=\bar{\tau}_{i} \cdot S_{U_{i}}^{\bar{c}} \bmod n$ and produces the signature $\bar{\sigma}_{i}=\left(\bar{s}_{i}, \bar{c}\right) . \quad U_{i} \neq U_{l}$ stores $\overline{\mathcal{Z}}$ and $\bar{c}$ and broadcasts $m_{i}^{\prime}=U_{i}\left\|X_{i}^{\prime}\right\| \bar{s}_{i}$. $U_{1}$ is assumed to be a trusted controller such that $U_{1}$ will be the last user to broadcast its message $m_{1}^{\prime}$ after all the other users have broadcast their messages $m_{j}^{\prime}$.

Authentication and Key Computation: Each $U_{i} \in G^{\prime}$ verifies the received messages $m_{j}^{\prime} \neq m_{i}^{\prime}$ by using the stored $\overline{\mathcal{Z}}$ and $\bar{c}$ to check the equation:

$$
\bar{c}=H\left(\left(\prod_{i=1}^{n} \bar{s}_{i}\right)^{e} \cdot\left(\prod_{i=1}^{n} H\left(U_{i}\right)\right)^{-\bar{c}}, \overline{\mathcal{Z}}\right)
$$

If equation (10) is correct, $U_{i}$ proceeds to verify the $X_{i}^{\prime}$ values using Lemma 1. If $\prod_{i=1, i \neq l}^{n} X_{i}^{\prime} \neq 1 \bmod p$, it means that at least one of the $X_{i}^{\prime}$ is incorrect. Then, all members will retransmit again. If each $U_{i} \in G^{\prime}$ correctly verifies that $\prod_{i=1, i \neq l}^{n} X_{i}=1 \bmod p$, then each 
$U_{i} \in G^{\prime}$ computes the new group key $K^{\prime}$ as follows:

$$
K^{\prime}=\prod_{i=1, i \neq l}^{n} g^{r_{i} r_{i+1}}=g^{r_{1}^{\prime} r_{2}+\cdots+r_{n} r_{1}^{\prime}} \bmod p(11)
$$

\section{Partition Protocol}

A partition can be seen as multiple users leaving the group. Let $G=\left\{U_{1}, \cdots, U_{n}\right\}$ be the current group, $\mathcal{L}$ be the group of partitioned/leaving users with the total number of partitioned/leaving users as $\ell_{d}$ and $G^{\prime}=G \backslash \mathcal{L}$ be the new group containing the remaining users. The Partition Protocol consists of two rounds and works as follows:

$\underline{\text { Round } 1}$ :All remaining odd-indexed users $U_{j} \in G^{\prime}$ for $j \in\{1,3,5, \cdots\}$ select new randoms $r_{j}^{\prime} \in Z_{q}^{*}$ and $\hat{\tau}_{j} \in$ $Z_{n}^{*}$ and computes $z_{j}^{\prime}=g^{r_{j}^{\prime}} \bmod p$ and $t_{j}^{\prime}=\hat{\tau}_{j}^{e} \bmod n$. $U_{j}$ then broadcasts $m_{j}=U_{j}\left\|z_{j}^{\prime}\right\| t_{j}^{\prime}$.

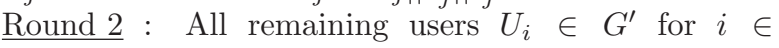
$\{1, \cdots, n\}$ compute $X_{i}^{\prime}$ using the same method as mentioned in Round 2 of the Leave Protocol.

Next, each remaining user $U_{i} \in G^{\prime}$ computes $\hat{\mathcal{Z}}=$ $\prod_{i=1}^{n} z_{i} \bmod p, \hat{\mathcal{T}}=\prod_{i=1}^{n} t_{i} \bmod n\left(\right.$ where $z_{i}=z_{j}^{\prime}$ and $t_{i}=t_{j}^{\prime}$ for remaining odd-indexed users $U_{j} \in G^{\prime}$ ) and $\hat{c}=H(\hat{\mathcal{T}}, \hat{\mathcal{Z}}) . \quad U_{i} \in G^{\prime}$ then computes $\hat{s}_{i}=$ $\hat{\tau}_{i} \cdot S_{U_{i}}^{\hat{c}} \bmod n$ and produces the signature $\hat{\sigma}_{i}=\left(\hat{s}_{i}, \hat{c}\right)$. $U_{i} \in G^{\prime}$ stores $\hat{\mathcal{Z}}$ and $\hat{c}$ and broadcasts $m_{i}^{\prime}=U_{i}\left\|X_{i}^{\prime}\right\| \hat{s}_{i}$. $U_{1}$ is assumed to be a trusted controller such that $U_{1}$ will be the last user to broadcast its message $m_{1}^{\prime}$ after all the other users have broadcast their messages $m_{j}^{\prime}$. Authentication and Key Computation: Each $U_{i} \in G^{\prime}$ verifies the received messages $m_{j}^{\prime} \neq m_{i}^{\prime}$ by using the stored $\hat{\mathcal{Z}}$ and $\hat{c}$ to check the equation:

$$
\hat{c}=H\left(\left(\prod_{i=1}^{n} \hat{s}_{i}\right)^{e} \cdot\left(\prod_{i=1}^{n} H\left(U_{i}\right)\right)^{-\hat{c}}, \hat{\mathcal{Z}}\right)
$$

If equation (12) is correct, $U_{i}$ proceeds to verify the $X_{i}^{\prime}$ values using Lemma 1 . If $\prod_{i=1, i \notin \mathcal{L}}^{n} X_{i}^{\prime} \neq 1 \bmod p$, it means that at least one of the $X_{i}^{\prime}$ is incorrect. Then, all members will retransmit again. If each $U_{i} \in G^{\prime}$ correctly verifies that $\prod_{i=1, i \notin \mathcal{L}}^{n} X_{i}=1 \bmod p$, then $U_{i}$ computes the new group key $K^{\prime}$ as follows:

$$
K^{\prime}=\prod_{i=1, i \notin \mathcal{L}}^{n} g^{r_{i} r_{i+1}}=g^{r_{1}^{\prime} r_{2}+\cdots+r_{n} r_{1}^{\prime}} \bmod p(13)
$$

\section{Complexity and Energy Analysis}

\section{Complexity Analysis}

We compare our dynamic protocols with the authenticated BD protocol using the efficient certificate-based ECDSA signature scheme. As mentioned earlier, the
$\mathrm{BD}$ protocol is re-executed whenever dynamic group membership events occur as no dynamic protocols have been specified by Burmester and Desmedt in [2]. The complexity of the $\mathrm{BD}$ protocol is based on the theoretical evaluation presented in [1] and [10]. The current group size, merging users, merging groups, leaving users, remaining odd-indexed users and height of key tree are denoted as $n, m, k, \ell_{d}, v$ and $h$ respectively.

\section{Table 4. Complexity Analysis of Dynamic Pro-} tocols

\begin{tabular}{||c|c|c|c|c|c|c||}
\hline \hline \multirow{2}{*}{ Protocol } & \multicolumn{2}{|c|}{ Comm Cost } & \multicolumn{3}{|c||}{ Comp Cost } \\
\cline { 2 - 7 } & $\mathrm{Rd}$ & Msgs & Exp. & $\begin{array}{c}\text { sign. } \\
\text { Gen. }\end{array}$ & $\begin{array}{c}\text { sign. } \\
\text { verif. }\end{array}$ \\
\hline \hline $\begin{array}{c}\text { BD } \\
{[2]}\end{array}$ & $\mathrm{J}$ & 2 & $2 n+2$ & $3^{a}$ & 2 & $n+3$ \\
\cline { 2 - 7 } & $\mathrm{L}$ & 2 & $2 n-2$ & $3^{a}$ & 2 & $n+1$ \\
\cline { 2 - 7 } & $\mathrm{M}$ & 2 & $2 n+2 m$ & $3^{a}$ & 2 & $\begin{array}{c}n+m \\
+2\end{array}$ \\
\cline { 2 - 7 } & $\mathrm{P}$ & 2 & $2 n-2 l_{d}$ & $3^{a}$ & 2 & $\begin{array}{c}n-l_{d} \\
+2\end{array}$ \\
\hline \hline \multirow{3}{*}{$\begin{array}{c}\text { Prop. } \\
\text { Sch. }\end{array}$} & $\mathrm{J}$ & 3 & 5 & $2^{b}$ & 1 & 1 \\
\cline { 2 - 7 } & $\mathrm{L}$ & 2 & $v+n-2$ & $3^{c}$ & 1 & 1 \\
\cline { 2 - 7 } & $\mathrm{M}$ & 3 & $6(k-1)$ & $4^{d}$ & 1 & 1 \\
\cline { 2 - 7 } & $\mathrm{P}$ & 2 & $v+n$ & $3^{c}$ & 1 & 1 \\
\hline \hline
\end{tabular}

${ }^{a}$ : All users in BD perform 3 exponentiations.

${ }^{b}$ : Only $U_{1}$ and $U_{n+1}$ perform 2 exponentiations each.

${ }^{c}$ : Only users $U_{j}$ for $j$ is odd perform 3 exponentiations and the rest performs 2 exponentiations each.

${ }^{d}$ : Only $U_{1}$ and $U_{n+1}$ perform 4 exponentiations each.

$\underline{\text { Discussion }}$

Although our Join and Merge Protocols require 1 extra round than the BD Join and Merge Protocol, however both our Join and Merge Protocols require fewer communication messages than the BD Join and Merge Protocols. For exponentiation cost, all users in the BD perform 3 exponentiations each for Join, Leave, Merge and Partition Protocols whereas for our Join Protocol, only $U_{1}$ and $U_{n+1}$ performs 2 exponentiations each while the rest need not perform any exponentiations. For our Merge Protocol, only $U_{1}$ and $U_{n+1}$ perform 4 exponentiations each while all other users need not perform any exponentiations. Our Leave and Partition Protocols require only users $U_{j} \notin \mathcal{L}$ for $j$ is odd to perform 3 exponentiations while $U_{k} \notin \mathcal{L}$ for $k$ is even to perform 2 exponentiations. All our dynamic protocols are also more efficient than the BD in terms of signature generation and verification.

\section{Energy Cost Analysis}

In this section, we present the energy cost analy- 
sis of our dynamic protocols and compare them with re-executing the authenticated $\mathrm{BD}$ protocol using the ECDSA signature scheme. We assume that each node in the group is using the $133 \mathrm{MHz}$ "StrongARM" microprocessor and the communication module used is the Spectrum24 WLAN card as presented in Table 2 and 3 respectively. We also assume that the current group size $n=100$, the number of merging users $m=20$ and the number of leaving users $\ell_{d}=20$. The energy cost results are presented in Table 5 .

Table 5. Energy Cost for Dynamic Protocols

\begin{tabular}{|c|c|c|}
\hline & & Energy \\
\hline \multirow{2}{*}{$\begin{array}{l}\text { BD } \\
\text { Join }\end{array}$} & $U_{1}-U_{n}$ & $1.234 J$ \\
\hline & $U_{n+1}$ & $2.31 J$ \\
\hline \multirow{4}{*}{$\begin{array}{c}\text { Our } \\
\text { Join } \\
\text { Protocol }\end{array}$} & $U_{1}$ & $0.039 \mathrm{~J}$ \\
\hline & $U_{n}$ & $0.049 \mathrm{~J}$ \\
\hline & $U_{n+1}$ & $0.057 J$ \\
\hline & Others & $1.34 \mathrm{~mJ}$ \\
\hline BD Leave & Remain. Users & $1.179 \mathrm{~J}$ \\
\hline \multirow{2}{*}{$\begin{array}{c}\text { Our } \\
\text { Leave Protocol }\end{array}$} & $U_{j}, j=$ odd & $0.160 \mathrm{~J}$ \\
\hline & $U_{k}, k=$ even & $0.150 \mathrm{~J}$ \\
\hline \multirow{2}{*}{$\begin{array}{c}\text { BD } \\
\text { Merge }\end{array}$} & Group A Users & $1.660 \mathrm{~J}$ \\
\hline & Group B Users & $2.532 J$ \\
\hline \multirow{3}{*}{$\begin{array}{c}\text { Our } \\
\text { Merge } \\
\text { Protocol }\end{array}$} & $U_{1}$ & $0.079 \mathrm{~J}$ \\
\hline & $U_{n+1}$ & $0.079 \mathrm{~J}$ \\
\hline & Others & $0.986 \mathrm{~mJ}$ \\
\hline BD Partition & Remain. Users & $0.942 J$ \\
\hline \multirow{2}{*}{$\begin{array}{c}\text { Our } \\
\text { Partition Protocol }\end{array}$} & $U_{j}, j=$ odd & $0.142 J$ \\
\hline & $U_{k}, k=$ even & $0.132 J$ \\
\hline
\end{tabular}

Discussion

Based on the energy cost results shown in Table 5, it can be easily seen that our protocols are much more suitable to be implemented in wireless networks where dynamic group membership events occurs frequently. If the $\mathrm{BD}$ protocol were to be implemented, then the battery life of each node will be depleted more rapidly due to the high energy costs. On the other hand, our dynamic protocols consume much lower energy as shown in Table 5.

\section{Conclusion}

We present an energy-efficient initial GKA protocol and four authenticated dynamic GKA protocols that take advantage of the ID-based signature scheme. The complexity and energy cost analysis of our five protocols show that they are much more suitable for wireless networks. The security of our protocols is based on the security of the BD protocol and the GQ signature scheme, both of which have been proven to be secure. Due to the page limit, the detail security analysis of our protocols will be provided in the full version.

\section{References}

[1] Y. Amir, Y. Kim, C. Nita-Rotaru, and G. Tsudik. On the performance of group key agreement protocols. John Hopkins Univ., Center of Networking and Distributed Systems, TR CNDS-2001-5, 2001.

[2] M. Burmester and Y. Desmedt. A secure and efficient conference key distribution system. In Advances in Cryptography - Eurocrypt '94, volume 950 of LNCS, pages 275-286, 1995.

[3] D. W. Carman, P. S. Kruss, and B. J. Matt. Constraints and approaches for distributed sensor network security. NAI Labs TR \#00-010, Sept. 2000.

[4] S. Cho, J. Nam, S. Kim, and D. Won. An efficient dynamic group key agreement for low-power mobile devices. In ICCSA 2005, volume 3480 of $L N C S$, pages 498 - 507, Apr. 2005.

[5] L. C. Guillou and J. J. Quisquater. A "paradoxical" identity-based signature scheme resulting from zeroknowledge. In Advances in Cryptology - Crypto '88, volume 0403 of $L N C S$, pages 216-231, 1990.

[6] A. Hodjat and I. Verbauwhede. The energy cost of secrets in ad-hoc networks. In IEEE CAS Workshop on Wireless Communication and Networking, Sept. 2002.

[7] I. Ingemarsson, D. T. Tang, and C. K. Wong. A conference key distribution system. IEEE Transaction on Information Theory, 28(5):714 - 720, Sept. 1982.

[8] R. Karri and P. Mishra. Optimizing the energy consumed by secure wireless sessions: wireless transport layer security case study. Mobile Networks and Applications, 8(2):177 - 185, Apr. 2003.

[9] J. Katz and M. Yung. Scalable protocols for authenticated group key exchange. In Advances in Cryptography - Crypto'03, volume 2729 of LNCS, pages 110 125, 2003.

[10] Y. Kim, A. Perrig, and G. Tsudik. Tree-based group key agreement. ACM Transaction on Information and System Security, 7(1):60 - 96, Feb. 2004.

[11] MIRACL. Multiprecision integer and rational arithmetic c/c++ library. http://indigo.ie/ mscott/.

[12] S. Saeednia and R. Safavi-Naini. Efficient identitybased conference key distribution protocols. In Information Security and Privacy - 3rd Australasian Conference, volume 1438 of LNCS, pages 320-331, 1998.

[13] R. Sakai, K. Ohgishi, and M. Kasahara. Cryptosystems based on pairing. In SCIS 2000, 2000.

[14] N. Saxena, G. Tsudik, and J. H. Yi. Identity-based access control for ad hoc groups. In ICISC 2004, volume 3506 of $L N C S$, pages 362-379, 2005.

[15] M. Steiner, G. Tsudik, and M. Waidner. Key agreement in dynamic peer groups. IEEE Transactions on Parallel and Distributed Systems, 11(8):769 - 780, Aug. 2000. 